\title{
Physical Activity and Cognition in the Northern Manhattan Study
}

\author{
Joshua Z. Willey ${ }^{\mathrm{a}}$ Yeseon Park Moon ${ }^{\mathrm{a}}$ Rachel Ruder ${ }^{\mathrm{a}}$ Yuen K. Cheung ${ }^{\mathrm{b}}$ \\ Ralph L. Sacco ${ }^{d-f}$ Mitchell S.V. Elkind ${ }^{a, c}$ Clinton B. Wright ${ }^{d, g}$ \\ Departments of a Neurology, ${ }^{b}$ Biostatistics and ${ }^{\mathrm{c} E p i d e m i o l o g y, ~ C o l u m b i a ~ U n i v e r s i t y, ~ N e w ~ Y o r k, ~ N . Y ., ~ a n d ~}$ \\ Departments of ${ }^{d}$ Neurology, ${ }^{e}$ Epidemiology and f Human Genetics, and ${ }^{\mathrm{f}}$ Evelyn F. McKnight Brain Institute, \\ University of Miami, Miami, Fla., USA
}

\section{Key Words}

Physical activity $\cdot$ Cognition $\cdot$ Dementia

\begin{abstract}
Background: To test the hypothesis that leisure time physical activity (PA) is associated with cognitive status. Methods: We assessed cognition using the Mini-Mental Status Examination (MMSE) at enrollment and using the modified Telephone Interview for Cognitive Status (TICS-m) administered annually since 2001 in the Northern Manhattan Study. Baseline measures of leisure time PA were collected via in-person questionnaires. Total PA was categorized into 3 groups based on the metabolic equivalent (MET) score, a composite of total reported intensity and time. We used linear regression models to examine the association of PA with MMSE, and generalized estimating equations for change in TICS-m over time. Results: There were 3,298 stroke-free participants with MMSE data (mean MMSE $26.0 \pm 3.8$ ) and 2,279 with TICS-m scores available. Compared to no PA, those with the upper quartile of MET scores had greater baseline MMSE scores (adjusted $\beta=0.4, p=0.01$ ) but no association with change in TICS-m over time. There were interactions $(p<$ 0.05 ) between PA and both insurance and education; compared to no PA, those in the upper quartile of MET scores had a greater MMSE score only among those with Medicaid/no
\end{abstract}

insurance (adjusted $\beta=0.83, p=0.0005)$ and those who did not complete high school (adjusted $\beta=0.68, p=0.001$ ). Conclusions: Increased levels of PA were associated with better baseline MMSE, particularly among those with socioeconomic disadvantages, but not with cognitive decline.

(c) 2013 S. Karger AG, Basel

\section{Introduction}

As the population continues to age, the public health impact of neurodegenerative conditions that present with cognitive impairment or dementia will be particularly high [1]. Several identified risk factors for cognitive impairment have shed light on the pathogenesis of the underlying causes, particularly on a genetic and molecular basis [2]. Physical activity (PA) is one useful target for prevention since individuals at risk may modify their behavior with little potential for adverse events or need for medications, and the added benefit of protection against other diseases of aging.

Several investigators [3-9], and a recent meta-analysis [10], have documented a dose-response association between baseline measures of PA and subsequent cognitive performance or risk of dementia; prior studies have demonstrated a protective effect on only vascular dementia,

\section{KARGER}

E-Mail karger@karger.com www.karger.com/ned (c) 2013 S. Karger AG, Basel

0251-5350/13/0422-0100\$38.00/0
Joshua Z. Willey, MD, MS

710 West 168th Street, Box 30

New York, NY 10032 (USA)

E-Mail jzw2@ columbia.edu 
or on Alzheimer disease alone [11, 12]. Not all groups, however, have documented an association between cognitive function and PA, particularly among older individuals $[6,13,14]$. Randomized clinical trials of PA programs have shown conflicting results [15-17]. A recent National Institutes of Health State-of-the-Science Conference Statement argued that there was probably a 'decreased risk' of cognitive decline with PA, though the data available was 'low quality' $[18,19]$. A recent Cochrane database review indicated insufficient evidence to support the effect of PA on cognition in older people [20]. Several unanswered questions remain. Few studies have used the same population to examine cognitive performance at baseline and changes over time. The populations studied have also varied, ranging from participants enrolled in middle adulthood to those over the age of 65 years; these studies have rarely enrolled participants with low socioeconomic status or educational achievement, or populations with a large proportion of Hispanics. Furthermore, few studies have examined whether the effect of PA could be modified by sociodemographics. We aimed to examine the independent association between measures of PA and cognitive performance at enrollment and over time and to examine whether baseline factors modified these associations. We hypothesized that total PA would be associated with higher Mini-Mental Status Examination (MMSE) performance at baseline, and a slower decline over repeated measures of the modified Telephone Interview for Cognitive Status (TICS-m).

\section{Methods}

Recruitment of the Cohort

The Northern Manhattan Study (NOMAS) is a populationbased prospective cohort study designed to evaluate the effects of medical, socioeconomic, and other risk factors on the incidence of stroke and other vascular outcomes in a stroke-free racially/ethnically diverse community cohort. The methods of participant recruitment, evaluation, and follow-up have been previously reported [21]. A total of 3,298 participants were recruited between 1993 and 2001, and participants were followed annually by telephone.

Standard Protocol Approvals, Registrations, and Patient

Consents

This study was approved by the Institutional Review Boards at Columbia University Medical Center and the University of Miami Miller School of Medicine. All participants provided written informed consent.

\section{Cohort Evaluation}

Data regarding baseline status and risk factors were collected through interviews of participants. Race-ethnicity was determined by self-identification. Standardized questions were asked regard- ing the following conditions: hypertension, diabetes, hypercholesterolemia, cigarette smoking, and cardiac conditions. Depression was defined based on a Hamilton Depression Rating Scale score $>10$ or a history of antidepressant use. Insurance status was defined as having no insurance or Medicaid versus having private insurance or Medicare [22]. Educational achievement was assessed via the self-reported grade at which school was completed and classified as completing high school versus not. We did not collect information regarding household income.

Cognitive performance at baseline was measured using the MMSE in English or a validated Spanish translation administered by trained bilingual research assistants.

Starting in 2001, a mean of 4.7 years after the initial MMSE, the TICS- $m$ was administered to participants during annual telephone follow-up [23]. The TICS- $m$ was designed to assess cognitive performance across multiple domains and includes calculations, delayed 10-word recall, language, and attention. It was administered over the phone at each annual follow-up and required $10 \mathrm{~min}$ on average to complete. Incomplete TICS- $m$ tests were not used in the statistical analysis as only the total score is valid.

\section{Assessment of PA}

PA was measured using an in-person questionnaire adapted from the National Health Interview Survey of the National Center for Health Statistics at enrollment, when the MMSE was also obtained [24]. This questionnaire records the duration and frequency of various leisure time/recreational activities for the 2 weeks prior to the interview. Participants were then asked if they had engaged in any PA in the preceding 2 weeks, and those who answered 'no' were coded as physically inactive. For each activity, the participants were asked the duration of activity and the number of times they engaged in this same activity, and if this level of activity was typical of other weeks. If the duration of activity was less than $10 \mathrm{~min}$, it was coded as 'no activity'. This questionnaire has been previously reported as reliable and valid in this population [25] and correlates with the body mass index (BMI) and activities of daily living. Objective measures of physical fitness correlate well with PA questionnaires [26].

Questionnaires were correlated with validated compendia of PA that outline the intensity of multiple leisure time PA measured in metabolic equivalents (MET) [27]. Total activity was summarized via the MET score, whereby the MET for each individual activity is multiplied by the frequency per week and the duration. Total energy expenditure was estimated by accounting for the participant's weight [21].

\section{Statistical Analysis}

Baseline demographics by PA categories were compared using the $\chi^{2}$ test for categorical variables and the Kruskal-Wallis test for continuous variables. We fitted: (1) linear regression models with MMSE as the outcome to calculate parameter estimates $(\beta)$ and $95 \% \mathrm{CI}$, and (2) generalized estimating equations with identity link to calculate parameter estimates $(\beta)$ and $95 \%$ CI for change in TICS-m scores over time.

The MET score was our primary exposure of interest and was categorized into 3 groups: (1) no PA (MET score $=0)$ as the reference (41\%), (2) intermediate PA (MET score $\leq 14$ ), and (3) high PA (MET score $>14$ ). In secondary analyses, PA was assessed as: (1) any versus none, and (2) total energy expenditure in increments of $500 \mathrm{kcal} /$ week. 
Table 1. Baseline demographics of the NOMAS participants $(n=3,298)$

\begin{tabular}{|c|c|c|c|c|c|}
\hline & $\begin{array}{l}\text { Whole } \\
\text { cohort } \\
(\mathrm{n}=3,298)\end{array}$ & $\begin{array}{l}\text { Physically } \\
\text { inactive } \\
(\mathrm{n}=1,347)\end{array}$ & $\begin{array}{l}\text { Intermediate } \\
\text { MET score } \\
(\mathrm{n}=1,178)\end{array}$ & $\begin{array}{l}\text { High } \\
\text { MET score } \\
(\mathrm{n}=773)\end{array}$ & $\begin{array}{l}p \text { value for } \\
\text { difference }^{d}\end{array}$ \\
\hline \multicolumn{6}{|l|}{ Sociodemographic characteristics } \\
\hline Age, years & $69.2(10.3)$ & $69.3(10.3)$ & $69.3(10.3)$ & $69.0(10.4)$ & 0.9 \\
\hline Women & $2,071(62.8 \%)$ & $898(66.7 \%)$ & $755(64.1 \%)$ & $418(54.1 \%)$ & $<0.0001$ \\
\hline \multicolumn{6}{|l|}{ Race-ethnicity } \\
\hline Non-Hispanic black & $803(24.4 \%)$ & $269(20.0 \%)$ & $319(27.1 \%)$ & $215(27.8 \%)$ & \multirow{2}{*}{$<0.0001$} \\
\hline Non-Hispanic white & $690(20.9 \%)$ & $212(15.7 \%)$ & $257(21.8 \%)$ & $221(28.6 \%)$ & \\
\hline Less than high school education & $1,786(54.2 \%)$ & $842(62.6 \%)$ & $614(52.1 \%)$ & $330(42.7 \%)$ & $<0.0001$ \\
\hline Medicaid or no insurance & $1,435(43.8 \%)$ & $688(51.3 \%)$ & $476(40.7 \%)$ & $271(35.4 \%)$ & $<0.0001$ \\
\hline \multicolumn{6}{|l|}{ Medical comorbidities } \\
\hline \multicolumn{6}{|l|}{ Alcohol use } \\
\hline Moderate use $\mathrm{a}^{\mathrm{a}}$ & $1,086(32.9 \%)$ & $371(27.5 \%)$ & $386(32.8 \%)$ & $329(42.6 \%)$ & $<0.0001$ \\
\hline Hypertension $^{\mathrm{b}}$ & $2,429(73.7 \%)$ & $1,018(75.6 \%)$ & $866(73.5 \%)$ & $545(70.5 \%)$ & 0.04 \\
\hline Diabetes mellitus ${ }^{\mathrm{c}}$ & $716(21.8 \%)$ & $325(24.2 \%)$ & $261(22.2 \%)$ & $130(16.8 \%)$ & 0.0003 \\
\hline High-density lipoprotein cholesterol & $46.8(14.6)$ & $45.6(14.1)$ & $47.0(14.9)$ & $48.4(14.8)$ & $<0.0001$ \\
\hline Any cardiac disease & $792(24.0 \%)$ & $329(24.4 \%)$ & $275(23.3 \%)$ & $188(24.3 \%)$ & 0.8 \\
\hline Depression & $336(10.2 \%)$ & $169(12.6 \%)$ & $107(9.1 \%)$ & $60(7.8 \%)$ & 0.0006 \\
\hline Taking cholesterol-lowering medications & $411(12.4 \%)$ & $179(13.3 \%)$ & $132(11.2 \%)$ & $100(12.9 \%)$ & 0.3 \\
\hline MMSE score at baseline & $26.0(3.8)$ & $25.4(4.2)$ & $26.2(3.5)$ & $26.7(3.2)$ & $<0.0001$ \\
\hline \multicolumn{6}{|c|}{$\begin{array}{l}\text { Values are presented as means } \pm \text { SD or numbers }(\%) \text {. } \\
\text { a Two or fewer servings of alcohol per day. } \\
\text { b Systolic blood pressure } \geq 140 \mathrm{~mm} \text { Hg or diastolic blood pressure } \geq 90 \mathrm{~mm} \text { Hg based on the average of } 2 \text { blood pressure measure- } \\
\text { ents, a physician's diagnosis of hypertension, or the patient's self-report of a history of hypertension or antihypertensive use. } \\
{ }^{c} \text { Fasting blood glucose } \geq 126 \mathrm{mg} / \mathrm{dl} \text {, the patient's self-report of diabetes mellitus, or insulin and/or hypoglycemic agent use. } \\
{ }^{\mathrm{d}} \chi^{2} \text { test for categorical variables and Kruskal-Wallis test for continuous variables. }\end{array}$} \\
\hline
\end{tabular}

Unadjusted and adjusted models were constructed with hypothesized confounders: demographics (age, sex, race-ethnicity, medical insurance, and education) and vascular risk factors (dyslipidemia, current tobacco use, moderate alcohol use, hypertension, depression, and diabetes). We tested for interactions between $\mathrm{PA}$ and baseline sociodemographic factors (age, sex, race-ethnicity, education, medical insurance), and stratified models were carried out only when the $\mathrm{p}$ value for the interaction term was $<0.05$. Final models were checked for improvements of fit using the likelihood ratio test (LRT) or the Wald test as appropriate. All analyses were conducted using SAS version 9.2 (Cary, N.C., USA).

\section{Results}

\section{Description of the Cohort}

The entire NOMAS cohort $(\mathrm{n}=3,298)$ contributed data on PA and MMSE at enrollment. The demograph- ics of the cohort are presented in table 1. The mean MMSE at baseline was 26 (median 27, interquartile range 24-29), with a lower value among those who did not complete high school (median 25, interquartile range 2228 ) versus those who did (median 28 , interquartile range 27-29), and those with Medicaid or no insurance (median 26, interquartile range 23-28) compared to their counterparts (median 28, interquartile range 26-29). The TICS-m was obtained annually in 2,279 participants after a mean follow-up of 4.7 years from baseline, and 1,969 participants had at least 2 measures (median 4; minimum 1 and maximum 9). Since we were primarily interested in the effect of PA on cognition in a community-based sample, we excluded 44 participants with stroke prior to the first TICS-m, as well as those with TICS- $\mathrm{m}$ administered after a stroke $(\mathrm{n}=60)$. The main 
Table 2. Association between measures of physical activity and MMSE score in the NOMAS

\begin{tabular}{llll}
\hline & Unadjusted analysis & Model $1^{\mathrm{a}}$ & Model $2^{\mathrm{b}}$ \\
\hline MET score (high vs. no PA) & $1.37(0.17,<0.0001)$ & $0.52(0.15,0.0006)$ & $0.40(0.15,0.01)$ \\
MET score (intermediate vs. no PA) & $0.84(0.15,<0.0001)$ & $0.40(0.13,0.003)$ & $0.36(0.13,0.007)$ \\
Total intensity of PA (/500 kcal/week) & $0.07(0.01,<0.0001)$ & $0.01(0.01,0.2)$ & $0.005(0.01,0.6)$ \\
Any PA vs. none & $1.05(0.13,<0.0001)$ & $0.44(0.19,0.0002)$ & $0.38(0.12,0.002)$ \\
\hline
\end{tabular}

Values are presented as $\beta$ (standard error, $\mathrm{p}$ value). ${ }^{\mathrm{a}}$ Adjusted for age, race-ethnicity, sex, insurance (Medicaid/none vs. others), and completing high school education. ${ }^{\mathrm{b}}$ Further adjusted for high-density lipoprotein cholesterol, current tobacco use, moderate alcohol use, hypertension, current cholesterol lowering medications, depression, and diabetes.

Table 3. Association between measures of PA and TICS-m over time in the NOMAS

\begin{tabular}{llll}
\hline & Unadjusted analysis & Model 1 & Model $2^{\mathrm{b}}$ \\
\hline MET score (high vs. no PA) & $-0.03 /$ year $(0.05,0.6)$ & $-0.03 /$ year $(0.05,0.6)$ & $-0.03 /$ year $(0.05,0.5)$ \\
MET score (intermediate vs. no PA) & $-0.02 /$ year $(0.05,0.6)$ & $-0.02 /$ year $(0.05,0.7)$ & $-0.02 /$ year $(0.05,0.7)$ \\
Total intensity of PA (/500 kcal/week) & $-0.002 /$ year $(0.003,0.6)$ & $-0.001 /$ year $(0.003,0.7)$ & $-0.001 /$ year $(0.003,0.8)$ \\
Any PA vs. none & $-0.02 /$ year $(0.04,0.6)$ & $-0.03 /$ year $(0.04,0.6)$ & $-0.02 /$ year $(0.04,0.6)$ \\
\hline
\end{tabular}

Values are presented as $\beta$ (standard error, $\mathrm{p}$ value). ${ }^{\mathrm{a}}$ Adjusted for age, race-ethnicity, sex, insurance (Medicaid/none vs. others), and completing high school education. ${ }^{b}$ Further adjusted for high-density lipoprotein cholesterol, current tobacco use, moderate alcohol use, hypertension, current cholesterol-lowering medications, depression, and diabetes.

reason for not obtaining TICS-m scores was death before the first TICS was administered $(n=525)$. Participants who did not have a TICS-m study were more likely to be younger, women, and Hispanic compared to white; participants were also less likely to have been smokers or have diabetes or coronary artery disease compared to the rest of the cohort.

\section{Association of PA and MMSE Using Linear \\ Regression}

PA was associated with the MMSE at enrollment (LRT with 2 d.f., $p=0.007$ ). In analyses adjusting for age, sex, race-ethnicity, insurance, and education (model 1), and further adjusting for vascular disease risk factors (model 2), those engaging in intermediate and high total PA had higher MMSE scores than those who were physically inactive (table 2). Total activity in kilocalories per week was only associated with MMSE in univariate analyses.

We found evidence that the effect of PA on MMSE differed by sociodemographics: health insurance status (LRT with 2 d.f., $p$ interaction $=0.03$ ) and educational achievement (LRT with 2 d.f., $\mathrm{p}$ interaction $=0.02$ ).
Compared to those who were inactive, those in the high PA group had higher MMSE scores if they had Medicaid or no insurance (adjusted $\beta=0.83$, standard error 0.24 , $\mathrm{p}=0.0005)$ or if they did not complete high school (adjusted $\beta=0.68$, standard error $0.21, \mathrm{p}=0.001)$. We found no associations among those with Medicare/private insurance or among those who completed high school.

\section{Association of PA and TICS-m}

The results of GEE models examining the association of PA with TICS-m over time are outlined in table 3. In summary, we found that PA was associated with TICS-m at the first measurement, but not with changes over time. In univariate analyses, 1 year of aging was associated with a decline in TICS-m of 0.01 per year $(\mathrm{p}<0.0001)$. Compared to those who were inactive, those with high PA had a trend towards greater TICS-m on the first assessment (adjusted $\beta=0.55$ points, $\mathrm{p}=0.06$ ). We did not find an association between PA and change in TICS-m performance over time ( $\chi^{2}$ with 2 d.f., $\mathrm{p}=0.83$ ). We found no interaction with insurance status, educational attainment, or baseline MMSE. 


\section{Discussion}

In our study we found that more leisure time PA was independently associated with better cognitive performance in a cross-sectional manner, but not over time. In further analyses we found that the associations between leisure time PA and performance on the MMSE were apparent only among those of lower socioeconomic status: i.e. those who did not complete high school or who had Medicaid or no insurance. PA remains an attractive target for preventing cognitive decline because it is relatively low risk, does not require medications or medical follow-up, and can be inexpensive. The mechanisms by which PA may influence the MMSE are several and can be categorized by vascular dependent and independent effects. PA modulates the effect and levels of stroke risk factors such as hypertension and diabetes [28], thereby potentially reducing the burden of subclinical cerebrovascular disease. PA may also lower the risk of subclinical cerebrovascular disease independently of its effects on modifiable stroke risk factors [22]. Extensive literature has demonstrated the protective effects of PA on the brain, including neurogenesis, angiogenesis, hippocampal size and levels of brain-derived neurotrophic factor [29], and neuronal connectivity [30]. We did not find an association with cognitive scores over time, counter to other studies [17]. Our results may differ for several reasons. We used the TICS-m as a global cognitive screening measure which may lack sensitivity for subtle changes in domains that have been previously associated with PA, such as cognitive speed and executive function. Further, we do not have information on prevalent or incident dementia in this sample, though the prevalence of dementia was less than $5 \%$ in a subsample of patients who underwent a full neuropsychological battery. The mean age of our participants was 71.5 years, which is younger than in most dementia studies, and our average of 4 years of followup may not have been long enough to detect an association. Close to one third of the sample died or had an incident cardiovascular event, the latter potentially a competing factor in cognitive decline. In our study we did not collect information on PA in earlier adulthood, which could be more protective [31]. Further, our data are observational and differed from randomized clinical trials of healthy participants, or those with mild cognitive impairment [15] or Alzheimer disease [32, 33]. Interestingly trials of PA in patients with Alzheimer's disease have mostly shown improvements in functional impairment and depression but not necessarily cogni- tion [34]. Lastly, it is possible that the influence of PA on cognition in later life is relatively weak, particularly in the face of other nonmodifiable risk factors such as age or genetics.

An additional novel finding in our study is that PA was associated with better performance on the MMSE only among those who have Medicaid/no insurance, and those who did not complete high school, while there were no associations among their counterparts. We did not collect information on household income but believe that these two measures could be a proxy for socioeconomic status. It may be that higher MMSE scores among those with better socioeconomic status and more education mitigate the effect of leisure time PA. Possibly only those at higher risk due to other factors would benefit from lifestyle interventions such as exercise. We may have been unable to detect an effect of PA in the upper ranges of the MMSE due to ceiling effects or lack of power.

Our study has important strengths, including an urban population with a large proportion of Hispanics and individuals with Medicaid or no insurance, who have been underrepresented in previous studies. We were able to examine the effects with both cross-sectional and longitudinal analyses to help investigate the concept of cognitive reserve [35]. Our study also has some important weaknesses, however. We did not have available direct measures of physical fitness or activity such actigraphy [36]. Actigraphy, however, only measures activity when the participant wears the device, and habitual activity may not be captured. In our study we did not collect TICS-m until 2001 and it was not measured in the entire cohort mostly due to early deaths. Cardiovascular disease was the main cause of death in our cohort, for which PA would be a protective factor. The missing TICS-m could lead to a nondifferential loss to follow-up, thereby biasing our results towards the null [37]. Overall in our cohort the TICS-m declined over time, and we have previously noted a deleterious effect of renal function on the TICS-m over time [38]; any independent effect of PA is likely to be clinically small if we are underpowered. We followed participants for a limited number of years, and it is possible that over a longer period of time there could be a protective effect of PA. One prior study showed a strong effect of PA when assessed in middle age, suggesting that by the time participants reach the average age in our study the underlying pathologic process leading to cognitive decline may already be underway without overt clinical signs. It is important to note that PA has protective effects against multiple oth- 
er conditions associated with aging, including stroke and MI $[21,39]$, and our findings should not discourage individuals from performing even light-intensity activities. As with any epidemiological study, it is not possible to establish causation and residual confounding may be present. Further studies will be required to clarify these causal pathways.

In our study we found that PA was associated with baseline cognitive status, particularly in older adults with a lower socioeconomic status, but it was not significantly associated with a decline in cognitive performance. This, however, should not dissuade older patients from exercising, but it highlights the importance of continuing to search for modifiable risk factors for cognitive decline.

\section{Acknowledgements and Funding}

Dr. Willey is supported by the National Institute of Neurological Disorders and Stroke (NIH/NINDS K23 NS 073104) and the National Institutes of Health Loan Repayment Program (NIH AG 30009). Dr. Wright is supported by National Institute of Neurological Disorders and Stroke (NIH/NINDS K02 NS 059729; NIH/ NHLBI R01 HL 108623) and the Evelyn F. McKnight Brain Institute. Funding for this project was provided by NIH/NINDS R37 NS 29993.

\section{Disclosure Statement}

The authors report no conflicts of interest.

\section{References}

$\checkmark 1$ Middleton LE, Yaffe K: Promising strategies 12 Abbott RD, White LR, Ross GW, Masaki KH, for the prevention of dementia. Arch Neurol 2009;66:1210-1215.

$\checkmark 2$ Cummings JL, Cole G: Alzheimer disease. JAMA 2002;287:2335-2338.

3 Etgen T, Sander D, Huntgeburth U, Poppert $\mathrm{H}$, Forstl H, Bickel H: Physical activity and incident cognitive impairment in elderly persons: the INVADE study. Arch Intern Med 2010;170:186-193.

4 Larson EB, Wang L, Bowen JD, et al: Exercise is associated with reduced risk for incident dementia among persons 65 years of age and older. Ann Intern Med 2006;144: 73-81.

5 Middleton LE, Manini TM, Simonsick EM, et al: Activity energy expenditure and incident cognitive impairment in older adults. Arch Intern Med 2011;171:1251-1257.

6 Rovio S, Kareholt I, Helkala EL, et al: Leisuretime physical activity at midlife and the risk of dementia and Alzheimer's disease. Lancet Neurol 2005;4:705-711.

7 Scarmeas N, Luchsinger JA, Schupf N, et al: Physical activity, diet, and risk of Alzheimer disease. JAMA 2009;302:627-637.

8 Vercambre MN, Grodstein F, Manson JE Stampfer MJ, Kang JH: Physical activity and cognition in women with vascular conditions. Arch Intern Med 2011;171:1244-1250.

-9 Verghese J, Lipton RB, Katz MJ, et al: Leisure activities and the risk of dementia in the elderly. N Engl J Med 2003;348:2508-2516.

10 Sofi F, Valecchi D, Bacci D, et al: Physical activity and risk of cognitive decline: a metaanalysis of prospective studies. J Intern Med 2011;269:107-117.

11 Ravaglia G, Forti P, Lucicesare A, et al: Physical activity and dementia risk in the elderly: findings from a prospective Italian study. Neurology 2008;70:1786-1794.

PA and Cognition in the NOMAS Curb JD, Petrovitch H: Walking and dementia in physically capable elderly men. JAMA 2004:292:1447-1453.

13 Podewils LJ, Guallar E, Kuller LH, et al: Physical activity, APOE genotype, and dementia risk: findings from the Cardiovascular Health Cognition Study. Am J Epidemiol 2005;161: 639-651.

14 Burns JM, Cronk BB, Anderson HS, et al: Cardiorespiratory fitness and brain atrophy in early Alzheimer disease. Neurology 2008;71: 210-216.

15 Baker LD, Frank LL, Foster-Schubert K, et al: Effects of aerobic exercise on mild cognitive impairment: a controlled trial. Arch Neurol 2010;67:71-79.

16 Kramer AF, Hahn S, Cohen NJ, et al: Ageing, fitness and neurocognitive function. Nature 1999;400:418-419.

17 Lautenschlager NT, Cox KL, Flicker L, et al: Effect of physical activity on cognitive function in older adults at risk for Alzheimer disease: a randomized trial. JAMA 2008;300: 1027-1037.

18 Plassman BL, Williams JW Jr, Burke JR, Holsinger T, Benjamin S: Systematic review: factors associated with risk for and possible prevention of cognitive decline in later life. Ann Intern Med 2010;153:182-193.

19 Daviglus ML, Bell CC, Berrettini W, et al: NIH State-of-the-Science Conference Statement: preventing Alzheimer's disease and cognitive decline. NIH Consens State Sci Statements 2010;27:1-30.

20 Angevaren M, Aufdemkampe G, Verhaar HJ, Aleman A, Vanhees L: Physical activity and enhanced fitness to improve cognitive function in older people without known cognitive impairment. Cochrane Database Syst Rev 2008;CD005381.
21 Willey JZ, Moon YP, Paik MC, Boden-Albala B, Sacco RL, Elkind MS: Physical activity and risk of ischemic stroke in the Northern Manhattan Study. Neurology 2009;73:17741779.

22 Willey JZ, Moon YP, Paik MC, et al: Lower prevalence of silent brain infarcts in the physically active: the Northern Manhattan Study. Neurology 2011;76:21122118.

23 de Jager CA, Budge MM, Clarke R: Utility of TICS-M for the assessment of cognitive function in older adults. Int J Geriatr Psychiatry 2003; 18:318-324.

24 Moss AJ, Parsons VL: Current estimates from the National Health Interview Survey: United States, 1985. Vital Health Stat 10 1986:i-iv, $1-182$.

25 Sacco RL, Gan R, Boden-Albala B, et al: Leisure-time physical activity and ischemic stroke risk: the Northern Manhattan Stroke Study. Stroke 1998;29:380-387.

-26 Siconolfi SF, Lasater TM, Snow RC, Carleton RA: Self-reported physical activity compared with maximal oxygen uptake. Am J Epidemiol 1985;122:101-105.

27 Ainsworth BE, Haskell WL, Whitt MC, et al: Compendium of physical activities: an update of activity codes and MET intensities. Med Sci Sports Exerc 2000;32:S498S504.

28 Thompson PD, Buchner D, Pina IL, et al: Exercise and physical activity in the prevention and treatment of atherosclerotic cardiovascular disease: a statement from the Council on Clinical Cardiology (Subcommittee on Exercise, Rehabilitation, and Prevention) and the Council on Nutrition, Physical Activity, and Metabolism (Subcommittee on Physical Activity). Circulation 2003;107: 3109-3116. 
29 Erickson KI, Voss MW, Prakash RS, et al: Exercise training increases size of hippocampus and improves memory. Proc Natl Acad Sci USA 2011;108:3017-3022.

-30 Cotman CW, Berchtold NC, Christie LA: Exercise builds brain health: key roles of growth factor cascades and inflammation. Trends Neurosci 2007;30:464-472.

>31 Middleton LE, Barnes DE, Lui LY, Yaffe K: Physical activity over the life course and its association with cognitive performance and impairment in old age. J Am Geriatr Soc 2010; 58:1322-1326.

32 Graessel E, Stemmer R, Eichenseer B, et al: Non-pharmacological, multicomponent group therapy in patients with degenerative dementia: a 12-month randomzied, controlled trial. BMC Med 2011;9:129.
33 Ahlskog JE, Geda YE, Graff-Radford NR, Petersen RC: Physical exercise as a preventive or disease-modifying treatment of dementia and brain aging. Mayo Clin Proc 2011;86:876884.

34 Teri L, Gibbons LE, McCurry SM, et al: Exercise plus behavioral management in patients with Alzheimer disease: a randomized controlled trial. JAMA 2003;290:20152022.

35 Stern Y: Cognitive reserve in ageing and $\mathrm{Al}-$ zheimer's disease. Lancet Neurol 2012;11: 1006-1012.
36 Buchman AS, Boyle PA, Yu L, Shah RC, Wilson RS, Bennett DA: Total daily physical activity and the risk of $\mathrm{AD}$ and cognitive decline in older adults. Neurology 2012;78:13231329.

37 Greenland S: Response and follow-up bias in cohort studies. Am J Epidemiol 1977;106: 184-187.

38 Khatri M, Nickolas T, Moon YP, et al: CKD associates with cognitive decline. J Am Soc Nephrol 2009;20:2427-2432.

39 Sacco RL, Khatri M, Rundek T, et al: Improving global vascular risk prediction with behavioral and anthropometric factors: the multiethnic NOMAS (Northern Manhattan Cohort Study). J Am Coll Cardiol 2009;54: 2303-2311. 